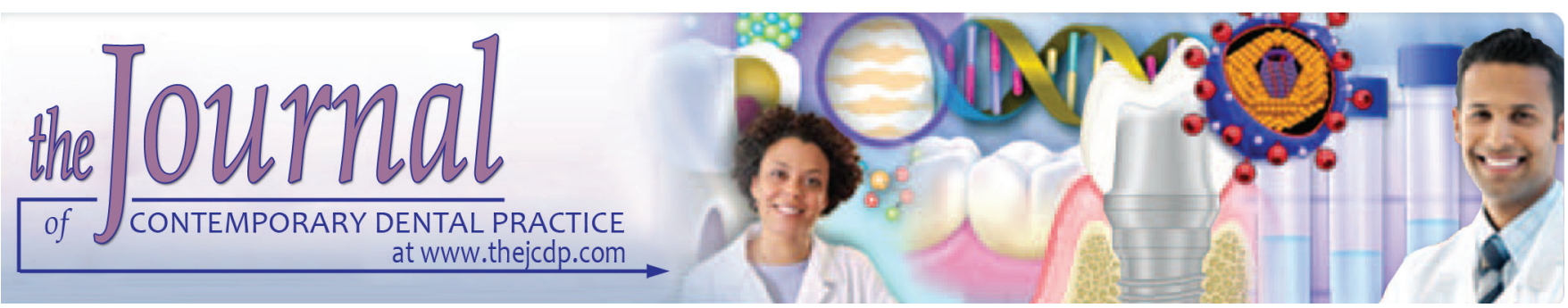

\title{
Biomarkers as Independent Predictors of Bone Regeneration around Biomaterials: A Systematic Review of Literature
}

\author{
${ }^{1}$ Sultan Albeshri, ${ }^{2}$ Abdulaziz Alblaihess, ${ }^{3}$ Abdurahman A Niazy, ${ }^{4}$ Sundar Ramalingam, ${ }^{5}$ Chalini Sundar \\ ${ }^{6}$ Hamdan S Alghamdi
}

\begin{abstract}
Background: Biomarkers are detected during bone formation and resorption associated with the dynamics of bone metabolism and are gaining importance as preferential indicators of bone healing in comparison with conventional methodologies. Current literature suggests that the usage of bone turnover markers for monitoring bone regeneration in association with biomaterials is limited.
\end{abstract}

Aim: To systematically review literature and evaluate whether bone-biomarkers can independently predict bone regeneration following implantation of various bone biomaterials.

Materials and methods: An electronic search was conducted in PubMed (MEDLINE) database from 1980 to January 2017. The articles for systematic review were selected based on formulated inclusion and exclusion criteria

Results: Upon database searching, 443 articles were retrieved and thoroughly reviewed based on the inclusion and exclusion

\footnotetext{
1,2Department of Periodontics and Community Dentistry, College of Dentistry, King Saud University, Riyadh, Kingdom of Saudi Arabia

${ }^{3}$ Molecular and Cell Biology Laboratory and Department of Diagnostic Dental Sciences, College of Dentistry, King Saud University, Riyadh, Kingdom of Saudi Arabia

${ }^{4}$ Department of Oral and Maxillofacial Surgery, College of Dentistry, King Saud University, Riyadh, Kingdom of Saudi Arabia

${ }^{5}$ Research Support Unit, College of Dentistry, King Saud University, Riyadh, Kingdom of Saudi Arabia

${ }^{6}$ Department of Periodontics and Community Dentistry, College of Dentistry, King Saud University, Riyadh, Kingdom of Saudi Arabia; Research Support Unit, College of Dentistry, King Saud University, Riyadh, Kingdom of Saudi Arabia

Corresponding Author: Hamdan S Alghamdi, Department of Periodontics and Community Dentistry, College of Dentistry, King Saud University, Riyadh, Kingdom of Saudi Arabia; Research Support Unit, College of Dentistry, King Saud University, Riyadh Kingdom of Saudi Arabia, Phone: +966114677732, e-mail: dalghamdi@ksu.edu.sa
}

criteria. In all, 41 studies were finally included for evaluation out of which 4 were clinical studies and the remaining 37 studies utilized animal models. On further evaluation, 12 studies reported the presence of biomarkers in association with cellular response during bone regeneration around biomaterials. Moreover, biomarkers related to enzyme activity and matrix protein derivatives were enhanced during bone-matrix deposition as reported in 14 studies. Inorganic skeletal matrix biomarkers indicative of bone mineralization showed positive expression in eight studies.

Conclusion: Several biomarkers appear to be useful for the assessment of bone regeneration around biomaterials. Although biomarkers are capable of independently predicting bone regeneration, lack of substantial evidence in the literature limits their true clinical utility.

Clinical significance: Noninvasive and inexpensive methods of isolating and characterization of biomarkers from cellular and extracellular skeletal matrix during bone regeneration have proven value in evaluating success of bone biomaterials.

Keywords: Biomarkers, Biomaterials, Bone regeneration, Systematic review.

How to cite this article: Albeshri S, Alblaihess A, Niazy AA Ramalingam S, Sundar C, Alghamdi HS. Biomarkers as Independent Predictors of Bone Regeneration around Biomaterials: A Systematic Review of Literature. J Contemp Dent Pract 2018;19(5):605-618.

Source of support: Nil

Conflict of interest: None

\section{BACKGROUND}

The prevalence of periodontal disease has increased and it has been recognized as the most common oral disease in recent times. It is characterized by periodontal infection followed by inflammation (periodontitis), leading to destruction of the supporting tooth, periodontal soft tissues, and the dental alveolar bone. The goal of 
periodontal therapy is to eliminate infection and inflammation, restore periodontal soft tissues, and stabilize the alveolar bone. ${ }^{1}$ A stable and healthy alveolar bone is necessary for the long-term functioning of dental implants (DI) and their corresponding superstructures. Guided tissue regeneration (GTR) and guided bone regeneration (GBR) aim to reconstruct periodontal soft tissues and regenerate damaged alveolar bone respectively, through the application of different biomaterials (i.e., membranes, bone substitutes) over an osseous defect. ${ }^{2}$ Guided bone regeneration is capable of regaining the contour of the diseased dental alveolar ridge ${ }^{3}$ and is also useful for socket preservation, ${ }^{4}$ thereby helping in the replacement of missing teeth with DI. ${ }^{5}$ As a result of its predictable benefits, GBR has become an integral part of periodontal therapy and DI rehabilitation procedures. ${ }^{6}$

In clinical practice, bone regeneration and healing are primarily evaluated by radiographic imaging in addition to bone sounding and histopathological evaluation of biopsied bone. ${ }^{7}$ There are several reported limitations to the traditional diagnostic methods which make optimal estimation of the success of GBR difficult. Moreover, radiographic determination of bone healing is highly subjective and can prove difficult to diagnose during the early phases of bone regeneration. ${ }^{8}$ The advent of bonebiomarkers as an assessment tool with the primary objective of monitoring early bone regeneration is therefore promising. Bone-biomarkers, when evaluated objectively, serve as an indicator of not only the normal bone healing process, but also pathogenic processes and responses to therapeutic intervention. Evidence-based literature acknowledges bone-biomarkers as a noninvasive, convenient, and relatively inexpensive indicator for monitoring bone metabolism and early healing. ${ }^{9}$

Bone-biomarkers indicative of metabolic processes include collagen breakdown products such as hydroxyproline, collagen crosslinks and telopeptides in addition to noncollagenous matrix proteins such as bone sialoprotein (BSP), osteoclast-specific enzyme like tartrate-resistant acid phosphatase (TRAP) and cathepsin K. On the contrary, biomarkers such as alkaline phosphatase (ALP), osteoblast-specific proteins like osteocalcin $(\mathrm{OCN})$ and osteopontin (OPN), and type I collagen (COL-1, byproduct of collagen neosynthesis) are secreted during different stages of bone formation. ${ }^{10}$ Similarly, bone-biomarkers are formed as byproducts of bone cell activity during the different phases of bone healing, ${ }^{11}$ which begins with early bone cell reactions, followed by bone matrix deposition, and, finally, matrix mineralization and remodeling. ${ }^{12}$

The utility of identifying bone-biomarkers during bone healing not only enhances the accuracy of assessing bone regeneration, but would also allow early detection of successful outcomes. ${ }^{13}$ Elevated levels of bone-biomarkers have been clinically detected in the serum and saliva, wherein their quantitative evaluation has proved to be of diagnostic and prognostic significance. ${ }^{14,15}$ In spite of their extensive clinical implications, only limited studies have demonstrated the utility of biomarkers as a diagnostic measure of bone regeneration. Even within the limited evidence available in the literature, biomarker evaluation has been considered only as a secondary tool of assessment of bone regeneration, while histopathology, histomorphometry, and radiographic imaging, or a combination of the above has remained the primary choice. Therefore, the objective of this systematic review of literature was to evaluate the role of bone-biomarkers in independently predicting bone regeneration following implantation of various biomaterials.

\section{MATERIALS AND METHODS}

\section{Focused Question}

The present review of the literature was conducted with the focused question: "Are bone-biomarkers capable of independently predicting bone regeneration following implantation of different bone biomaterials in an osseous defect?"

\section{Literature Search and Selection Criteria}

A systematic review of published studies evaluating bone-biomarkers during bone regeneration from 1980 until and including January 2017 was conducted. An electronic search was organized in PubMed (MEDLINE) database using the terms "bone-biomarkers," "bone regeneration," and "bone biomaterials" in combination with the Boolean operators "AND" and "OR."16 Following this, a manual search was performed additionally by screening the bibliographies of relevant retrieved articles and adding free-text words from titles or abstracts to identify potentially pertinent articles.

All articles retrieved through the literature search were imported into a bibliographic referencing software program (EndNote X7), and duplicate references were identified and removed. In order to eliminate selection bias, two independent reviewers (SA, AA), who were calibrated for intraobserver and interobserver reliability and agreement screened the relevant titles, abstracts, and full texts, and the articles for final review were selected according to preferred reporting items for systematic reviews and meta-analysis (PRISMA) guidelines, ${ }^{17}$ based on the following inclusion and exclusion criteria.

\section{Inclusion Criteria}

- Original research articles published in the English language, based on human clinical trials, case-control 
studies, cohort studies, case series, and case reports in addition to in vivo animal studies related to the usage of bone-biomarkers for evaluating bone regeneration in osseous defects following placement of biomaterials.

- Articles presenting data pertaining to the model used for research, biomaterials used for bone regeneration, bone-biomarkers evaluated, and the methods used for their assay, along with information relating to followup examination protocols.

\section{Exclusion Criteria}

Studies with insufficient information, ex vivo and in vitro researches, case reports, reviews and, technical and personal communications.

\section{Data Extraction and Study Characteristics}

Data extraction from all included studies was independently performed and verified by the two reviewers. When both reviewers agreed on exclusions, the reasons for exclusion were recorded. Any remaining disagreements were resolved by consensus or discussion, if necessary. The data extraction process was guided by a data extraction sheet that specified the relevant study characteristics, including author, year of publication, study design, information related to bone regeneration procedures (type of implanted biomaterials, anatomical site, and healing time), data for the biomarkers assessment (type and assay methods), and reported biomarkers' outcomes that evaluate bone formation in relation to the implanted biomaterials.

\section{RESULTS}

A total of 443 studies were identified through electronic searching of the PubMed (MEDLINE) database. Screening the titles and abstracts of the identified studies led to the selection of 117 full-text manuscripts, which were scrutinized and narrowed down to 62 studies, based on their relevance to the focused question of the present review. Following exclusion of duplicates, 41 studies fulfilled the inclusion criteria and were finally considered for systematic review (Flow Chart 1). The selected studies were reviewed by both the authors for the purpose of data extraction, and interpretation. A detailed characterization of the study objectives, study subjects (human/animal model), type of defect investigated along with implanted bone biomaterial, and the biomarkers evaluated under the specified time period for desirable outcomes is elaborated in Table 1.

\section{Description of Experimental Methods}

Majority of the reviewed studies used animal models in their research, while only four studies ${ }^{18-21}$ were based on
Flow Chart 1: Search strategy and articles included in the systematic review

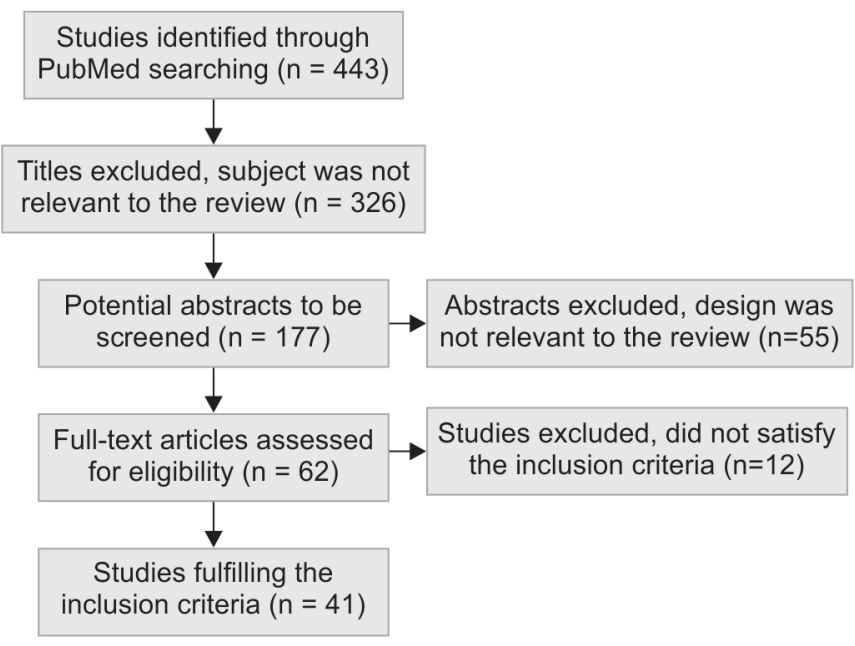

human clinical models. While two out of the four human studies, evaluated the role of biomarkers for assessment of bone regeneration, ${ }^{19,21}$ one of the studies was based on the assessment of bone formation along with $\mathrm{GTR}^{20}$ and another study was based on osseointegration and new bone formation around titanium DI. ${ }^{18}$ Heterogeneity in terms of the protocols of biomaterial implantation and their respective follow-up periods were widely observed among the reviewed studies. Interestingly, the shortest period of biomaterial implantation, which was evaluated, was 1 day, ${ }^{22,23}$ whereas the maximum period of biomaterial implantation was observed to be 16 weeks. ${ }^{18}$

Several bone biomaterials were investigated (e.g., different bone grafts and bone substitutes, membranes, titanium DI with surface modifications, and scaffolds loaded with drugs, osteogenic cells, or biological factors) in different studies. All the included studies reported some degree of bone regeneration based on the assessment of several biomarkers as mentioned in (Table 1). Heterogeneity was observed among the 37 animal studies, in terms of the anatomical sites chosen to recreate an osseous defect for placement of the biomaterials. Several studies were based on a rodent model, out of which one study in mice ${ }^{24}$ and another study in rats ${ }^{25}$ used maxillary defects. Similarly, mandibular defects were the chosen site in sheep, ${ }^{26}$ rabbit $^{27}$ and rat $^{28-33}$ models in eight of the included studies. While cranial and calvarial defects in rats were created in four of the reviewed experiments, ${ }^{34-37}$ two studies were based on calvarial ${ }^{22}$ and spinal ${ }^{38}$ defects in mice respectively. Among the other experimental models in rats, femur, ${ }^{39-41}$ ulna, ${ }^{42}$ tibia, ${ }^{40,43-45}$ and intramuscular sites ${ }^{46}$ were utilized for the evaluation of bone regeneration.

Nine of the reviewed studies examined the role of biomarkers, based on bone regeneration experiments in rabbit models. The osseous defect sites used in those 

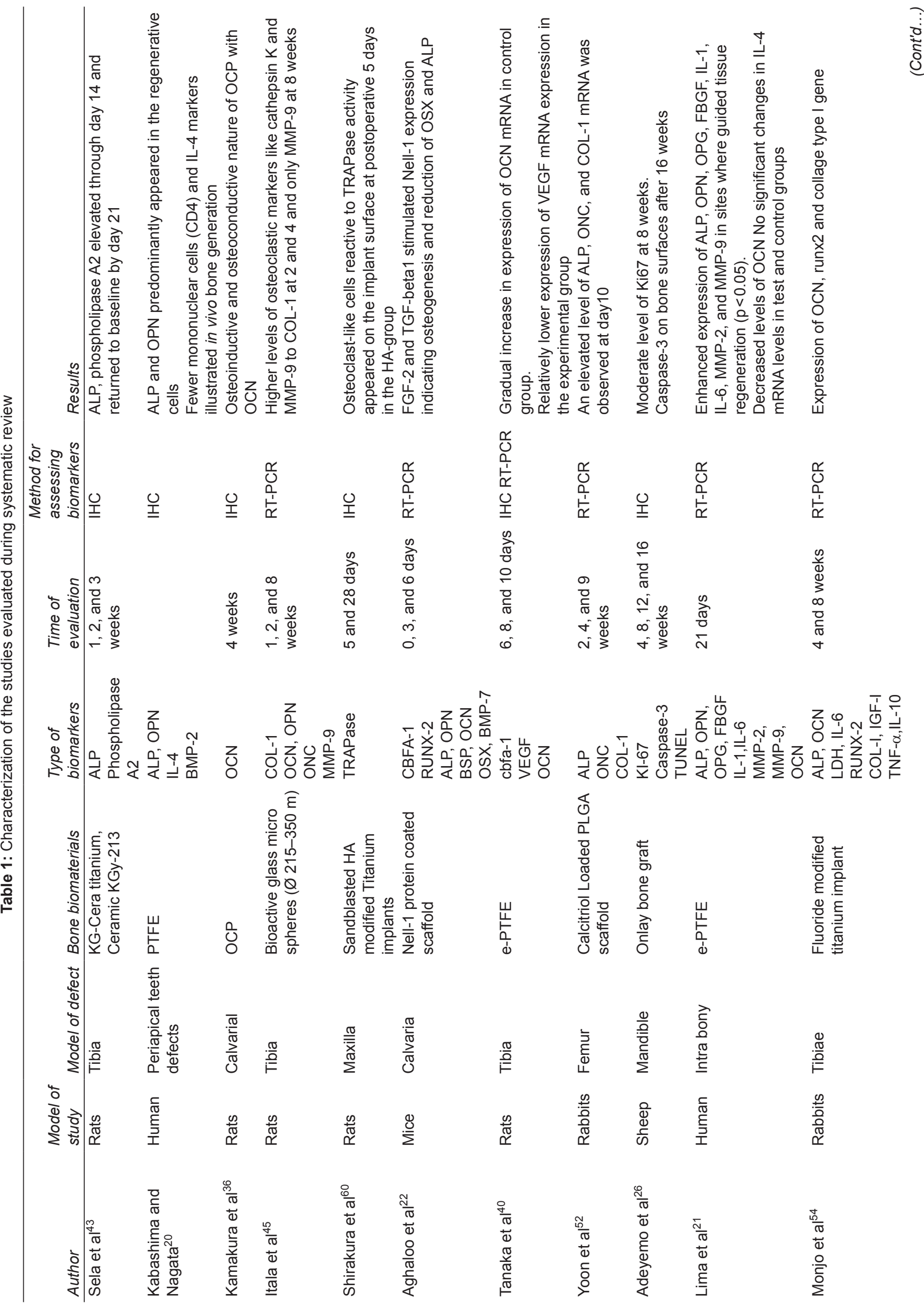


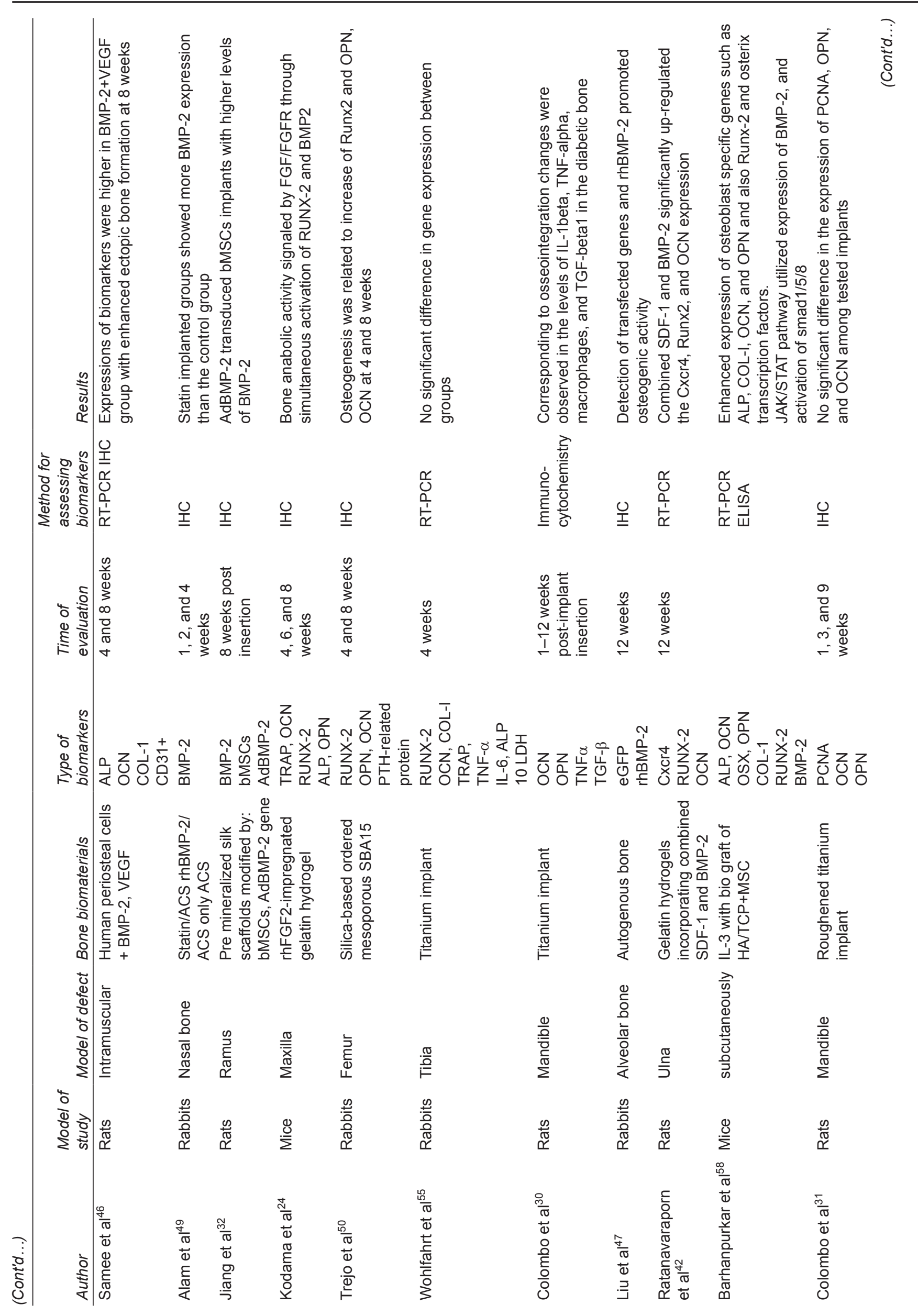




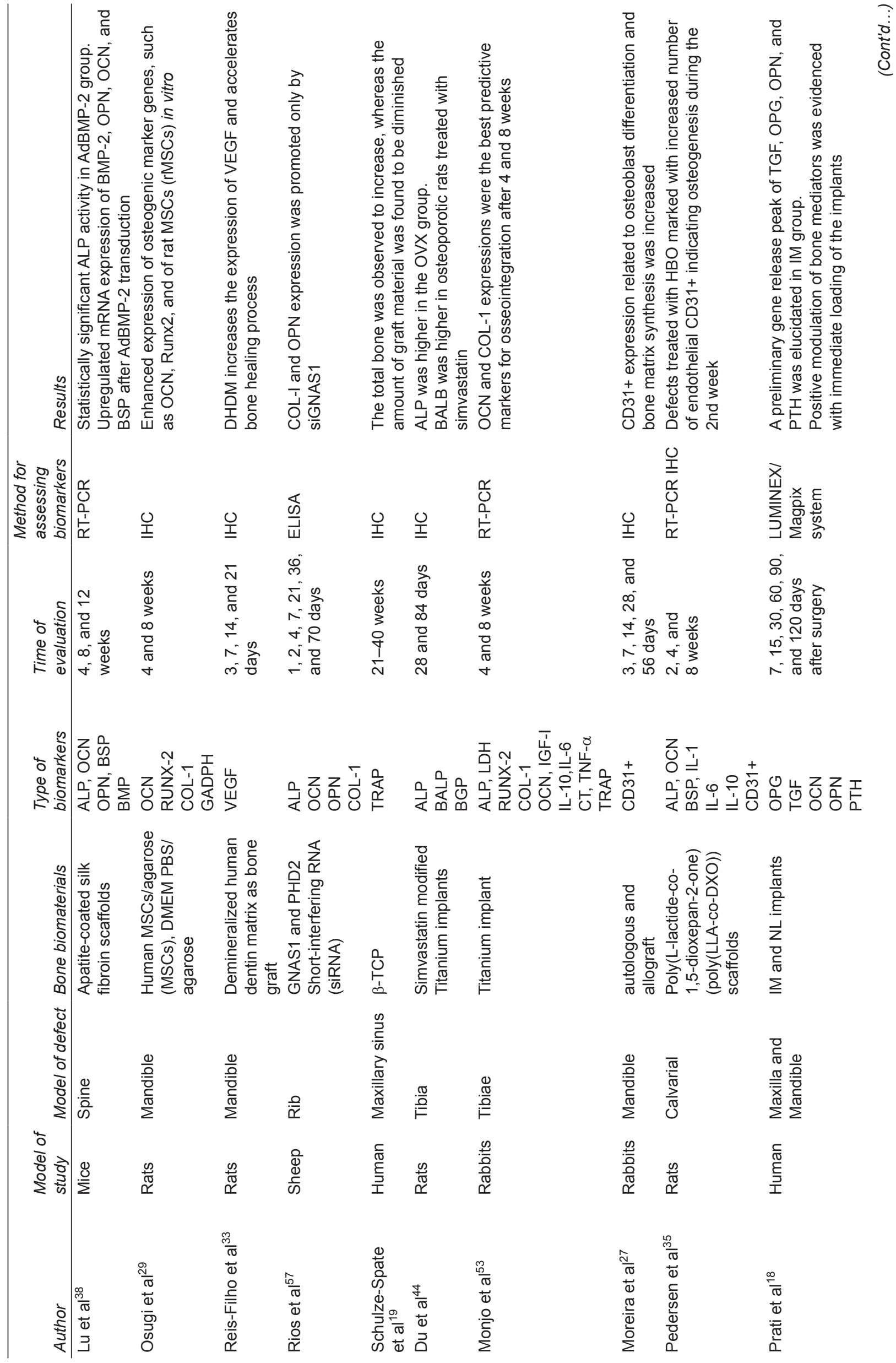




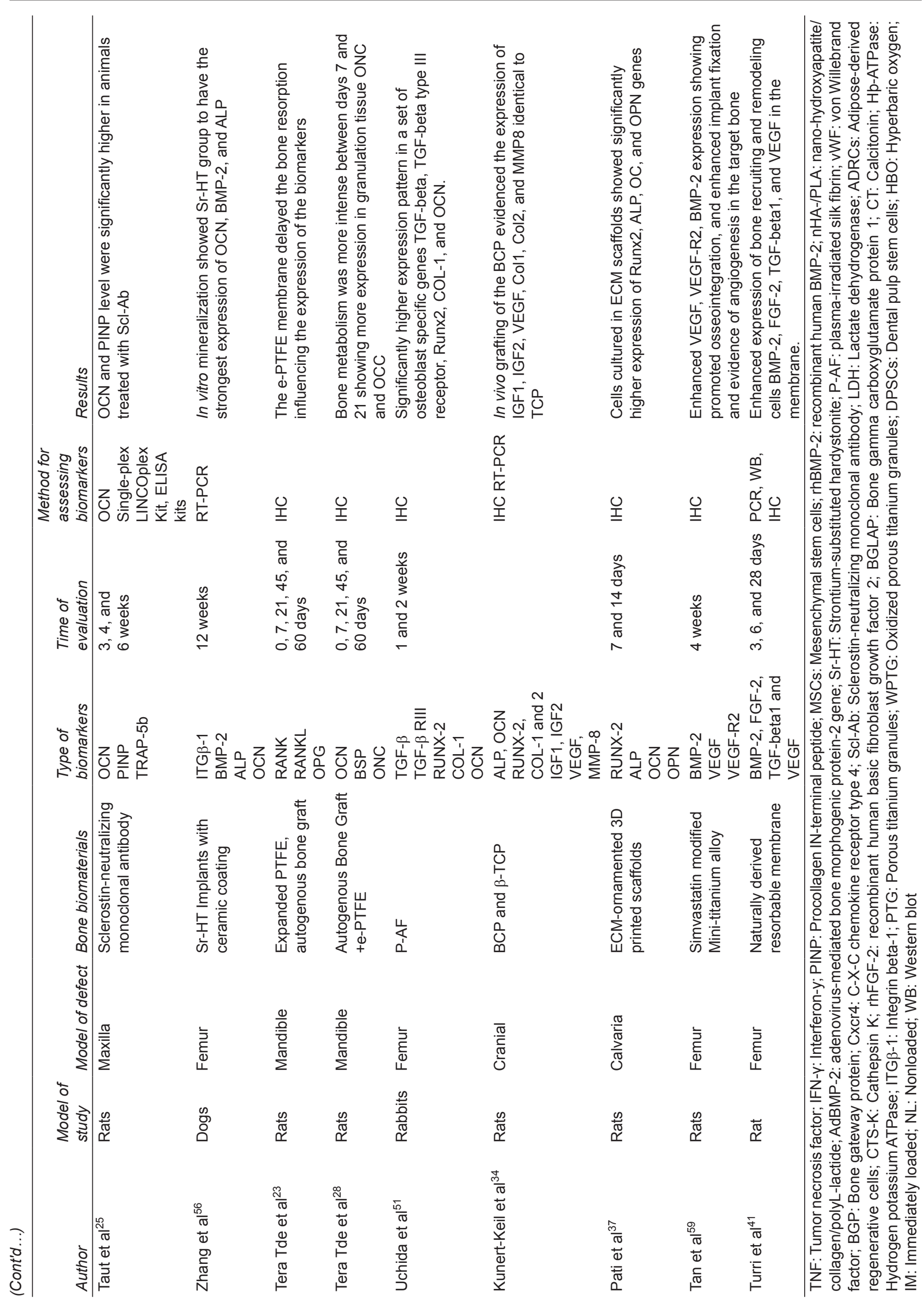


experiments included the alveolar bone, ${ }^{47}$ mandible, ${ }^{27,48}$ nasal bone, ${ }^{49}$ femur, $^{50-52}$ and tibia. ${ }^{53-55}$ Furthermore, in vivo osseointegration around titanium DI was evaluated in the canine femur, ${ }^{56}$ and in the mandible ${ }^{26}$ and the ribs $^{57}$ in a sheep model. The commonly used biomarker assays were immunohistochemistry (IHC) or reverse transcription polymerase chain reaction (RT-PCR) or a combination of both ${ }^{34,35,40,46}$ (Table 1). However, a few studies applied other methodologies such as multiplex bead array assay (LUMINEX) ${ }^{18,25}$ and enzyme-linked immunosorbent assay (ELISA) ${ }^{25,57,58}$ for analyzing and reporting their results.

\section{Outcomes based on Clinical Studies}

Schulze-Spate et $\mathrm{al}^{19}$ aimed to evaluate the biomarkers of healing process following bone augmentation in the maxillary sinus of patients using beta-tricalcium phosphate $(\beta$-TCP). They reported that TRAP staining was significantly associated with a decrease in grafted material and increase in new bone formation. Similarly, Kabashima and Nagata ${ }^{20}$ demonstrated interleukin (IL)4-producing cells to be associated with successful in vivo bone regeneration. Evaluating new bone formation and osseointegration around titanium DI, Prati et $\mathrm{al}^{18}$ reported the presence of transforming growth factors (TGFs), OCN, osteoprotegerin (OPG), OPN, and parathyroid hormone (PTH) during the early phase following implant placement and loading. Interestingly, progressively higher levels of bone-biomarker were recorded during the 7 th, 15 th, and 30th days, thereby indicating the validity of evaluating biomarkers as a surrogate predictor of the different phases of bone mineralization. ${ }^{18}$

\section{Outcomes based on Translational Studies}

Among the criteria evaluated for expression of bonebiomarkers in the reviewed studies based on translational animal models, the least degree of disparity was observed in terms of the types of biomaterials (bone grafts, titanium DI, 3D scaffolds, and other biological derivatives) used for bone regeneration and healing (Table 1). Therefore, further analysis of the data was accomplished based on this aspect of the reviewed studies.

\section{Studies using Bone-grafting Materials}

Fourteen out of the 41 studies investigated the presence of bone-biomarkers during the osteoinductive and osteoconductive phases of bone healing when autogenic, allogeneic, or other alloplastic bone substitutes were placed in osseous defect sites (Table 1). Moreira et $\mathrm{al}^{27}$ illustrated enhanced angiogenesis and expression of cluster of differentiation $31+(\mathrm{CD} 31+)$ associated with osteoblastic differentiation at 8 weeks, when using autogenic and allogeneic onlay bone grafts along with platelet-rich plasma (PRP). Interestingly, early osteoclastic activity demonstrated by OCN expression was followed by Ki-67, caspase-3, and the terminal deoxyribonucleotidyl transferase (TdT)-mediated biotin-16-dUTP nick-end labelling (TUNEL) expression, at 16 weeks, also with onlay bone grafts. ${ }^{26}$ Three of the reviewed animal experiments ${ }^{23,28,40}$ showed that expanded polytetrafluoroethylene (e-PTFE) membrane, when used along with autogenous bone, delayed resorption of the grafted bone, and histologically exhibited greater expression of OCN, BSP, osteonectin (ONC), OPG, RANK, and receptor activator of nuclear factor (NF)- $\mathrm{B}$ ligand (RANKL), while expressing relatively lower quantities of vascular endothelial growth factor (VEGF) and core binding factor alpha-1 (CBFA-1), possibly attributable to the high remodeling rate.

Interestingly, one of the studies ${ }^{20}$ on human dental periapical defects with GTR demonstrated ALP and OCN in fibroblasts-like regenerative cells and IL-4 in adjacent tissues of proven bone regeneration. Similarly, demineralized human dentin matrix (DHDM) ${ }^{33}$ represented an efficient grafting material for bone regeneration with increased expression of VEGF and accelerated bone healing. The functional efficacy of the calcium phosphatebased bone substitutes was evaluated using biomarkers in five of the included studies. Bone defect sites grafted with osteoconductive scaffolds like biphasic calcium phosphate $(\mathrm{BCP})$, octa-calcium phosphate $(\mathrm{OCP})^{36}$ and $\beta$-TCP evidenced an increase in the total bone volume ${ }^{34}$ and predominantly expressed ALP, OCN, Runt-related transcription factor 2 (RUNX-2), phosphate-regulating neutral endopeptidase, $X$-linked (PHEX), collagen (COL-1 and 2), insulin-like growth factor (IGF-1), IGF-2, VEGF, matrix metalloproteinase-8 (MMP-8), and bone morphogenetic protein (BMP). While a dose-dependent increase in osteoblast differentiation and matrix mineralization was observed in IL-3 impregnated mesenchymal stem cells grafted along with hydroxyapatite (HA)/TCP, 58 TRAP-positive osteoclast-like cells ${ }^{19}$ and osteoclastic markers cathepsin K and MMP- $9{ }^{45}$ were reported on the surfaces of HA, $\beta$-TCP, and bioactive glass scaffolds.

\section{Studies evaluating Bone Regeneration around Titanium Dls}

The clinical success of titanium DI for esthetic and functional rehabilitation can be substantiated with evidence at the molecular level wherein biomarkers of osseointegration such as OCN and COL- $1^{30,53,59}$ have been illustrated during periods ranging from 4 to 8 weeks post-implant placement. In a study based on fluoride-coated titanium DI, ${ }^{54}$ the expression of OCN, RUNX-2, and COL-1 correlated with modulatory effects of fluoride upon bone formation/resorption phases at the bone-implant biological 
interface. Similarly, simvastatin coating around DI placed in an osteoporotic rat model resulted in enhanced angiogenesis and osseointegration as evidenced by the increased expression of VEGF and bone ALP respectively. ${ }^{44,59}$ In contrast, no significant changes in biomarker expression were observed in titanium DI with surface modifications comprising of porous titanium granules, ${ }^{55}$ roughening, ${ }^{31}$ and TCP/HA coating. ${ }^{31}$ Nevertheless, ALP, VEGF-R2, CD31, RUNX-2, OCN, COL-1, TRAP, IL-6, TNF- $\alpha$, bone alkaline phosphatase (BALP), OPN, and OCN were commonly expressed during osseointegration, thereby indicating their significance in prognostic and clinical performance assessment. On the contrary, bone healing around DI showed an increased matrix vesicle enzyme activity (phospholipase-2) with bone bonding material like KG cera than nonbonding material like KGy-213. ${ }^{43}$ However, enhanced or delayed mineralization correlated with the expression of ALP. In a similar study on rat maxilla, ${ }^{60}$ increased TRAPase activity was reported for sandblasted DI with ceramic coating. Nonetheless, strontium-substituted hardystonite ceramic coating structure had the strongest expression of OCN, BMP-2, and ALP with increased osseointegration ability in comparison with other ceramic-coated DI. ${ }^{56}$

\section{Studies evaluating Bone Regeneration in Three-dimensional Scaffolds}

Encapsulation of bioactive molecules in three-dimensionally (3D) engineered scaffolds not only provides mechanical competence during bone regeneration, but was also coherent with elevated levels of biomarkers such as ALP, ONC, BMP, BSP, RUNX-2, and OPN. 22,32,35,37,38,52 More specifically, calcitriol(1,25[OH]2D3)-loaded porous poly(D,L-lactide-co-glycolide) (PLGA) scaffolds ${ }^{52}$ along with mesenchymal stem cells, when used for treating large bone defects, resulted in expression of COL-1 in addition to other biomarkers. Similarly, apatite-coated silk fibroin scaffolds, ${ }^{38}$ when tested for ectopic new bone formation, revealed upregulated expression of BMP-2, OPN, OCN, and BSP. Additionally, scaffolds of extracellular matrix (ECM), ${ }^{37}$ copolymers of poly(L-lactide-co1,5-dioxepan-2-one), ${ }^{35}$ premineralized silk along with BMP-2 modified bMSCs, ${ }^{32}$ simvastatin loaded atelocollagen sponge (ACS), ${ }^{49}$ and Nell-1 protein coat ${ }^{22}$ led to enhanced bone formation with significant expression of ALP, OCN, BMP, BSP, IL-1, IL-6, IL-10, CD31, RUNX-2, and OPN.

\section{Studies evaluating Bone Formation using Other Proteins and Cell Derivatives}

Taut et $\mathrm{al}^{25}$ assessed the positive therapeutic potential of sclerostin antibody ( $\mathrm{Scl}-\mathrm{Ab})$ to stimulate alveolar bone regeneration in rats demonstrating concurrent expression of higher levels of OCN and procollagen type I N propeptide (PINP) in sclerostin-neutralizing monoclonal antibody (Scl-Ab) treatment groups. Under similar conditions, plasma-irradiated silk fibrin ${ }^{51}$ and osteostatinloaded silica-based mesoporous SBA15 materials ${ }^{50}$ grafted in the rabbit femur resulted in significantly higher expression of TGF- $\beta$, TGF- $\beta$ RIII, RUNX-2, COL- 1 , and OCN owing to new bone formation. Remarkable bone growth was evidenced in osseous defect sites regenerated with VEGF-transfected mesenchymal stem cells and BMP-2 leading to enhanced expression of ALP, OCN, stromal cell-derived factor 1 (SDF-1), IL-6, COL-1, and CD31+. ${ }^{46}$ Similarly, gelatin hydrogel combined with SDF-1, BMP-2, ${ }^{42}$ and recombinant Fibroblast growth factor-2 (rhFGF2) ${ }^{24}$ led to optimized bone formation with fibroblast growth factor (FGF)/ fibroblast growth factor receptor (FGFR) signaled bone anabolic activity and simultaneous expression of RUNX-2 and BMP-2 biomarkers.

\section{DISCUSSION}

Bone is a metabolically active tissue and its regeneration comprises of well-orchestrated series of biological events. This continuous process of bone remodeling involves formation (osteoblasts), resorption (osteoclasts), and maintenance (osteocytes) in a definable and spatial sequence affected by intracellular and extracellular signaling pathways. ${ }^{61}$ Currently, there are a plethora of available bone augmentation strategies along with advanced cellular analytical methods for characterization of these bone-forming cells and identification of the transcriptional and translational profiles of genes and proteins encountered. ${ }^{11}$ Hence, molecular markers of bone have gained importance in recent times to detect the dynamics of bone during various phases of regeneration. ${ }^{9}$ In the present review, several studies demonstrated the efficacy of bone-biomarkers as prognostic indicators for the different stages of bone regeneration, in osseous defect sites, following placement of biomaterials. The commonly expressed biomarker identifiable during each stage of bone regeneration and healing when associated with biomaterials is elaborated in Table 2 .

\section{Biomarkers of Bone Turnover}

While biochemical indexes are capable of differentiating the biomarkers of bone formation and resorption, a sharp distinction may not be appreciated in clinical scenarios. This is clearly evident in the present systematic review wherein most OC fragments where detected in both matrix deposition and mineralization stages of bone healing $^{51,53,58}$ (Table 2). Similarly, BMP-2 and COL-1 $23,35,51$ 
Table 2: Bone-biomarkers related to bone regeneration process

\begin{tabular}{|c|c|c|}
\hline $\begin{array}{l}\text { Bone } \\
\text { regeneration } \\
\text { process }\end{array}$ & Related biomarkers & References \\
\hline $\begin{array}{l}\text { Early bone cell } \\
\text { reactions }\end{array}$ & $\begin{array}{l}\text { ALP, PCNA, } \\
\text { BMP-2, COL-1, } \\
\text { PCNA, OPN, } \\
\text { RUNX-2, CBFA-1 }\end{array}$ & $\begin{array}{l}\text { Rios et al }\left.\right|^{57} \\
\text { Pedersen et al } \\
\text { Barhanpurkar et al }^{58} \\
\text { Colombo et al } \\
\text { Marukawa et al }{ }^{48} \\
\text { Tanaka et al } \\
{ }^{40}\end{array}$ \\
\hline $\begin{array}{l}\text { Bone matrix } \\
\text { deposition }\end{array}$ & $\begin{array}{l}\text { OCN, COL-1, } \\
\text { BSP, OPN, } \\
\text { CD31+, OCN, } \\
\text { BMP-2 and 7, RUNX-2, } \\
\text { OSX, CBFA-1, } \\
\text { ONC, VEGF }\end{array}$ & $\begin{array}{l}\text { Uchida et } \mathrm{al}^{51} \\
\text { Pedersen et } \mathrm{al}^{35} \\
\text { Barhanpurkar et al } \\
\text { Tera Tde et } \mathrm{al}^{23,28} \\
\text { Colombo et } \mathrm{al}^{30} \\
\text { Tanaka et } \mathrm{al}^{40} \\
\text { Moreira et } \mathrm{al}^{27}\end{array}$ \\
\hline $\begin{array}{l}\text { Bone } \\
\text { mineralization }\end{array}$ & $\begin{array}{l}\text { OCN, OPN, } \\
\text { VEGF, CD31+, } \\
\text { Ki-67, Caspase-3, } \\
\text { TUNEL }\end{array}$ & 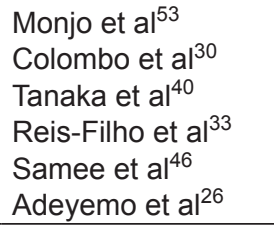 \\
\hline
\end{tabular}

were expressed during early cell reactions through matrix deposition phases. Moreover, several of the reported biomarkers of bone turnover could have resulted from the nonskeletal processes and might be present in other tissues influencing their circulating levels. ${ }^{33}$

In clinical practice, implantation of bone biomaterials within osseous defect sites is associated with high degrees of success in relation to bone regeneration and healing. ${ }^{6}$ Nevertheless, complications arise in 5 to $10 \%$ of patients, making them liable to failed bone regeneration and impaired bone healing. ${ }^{62}$ Such complications associated with bone biomaterials could be attributed to several factors including a characteristic of host bone, infected tissue, lack of blood supply, and disturbances to the stability of implanted biomaterials during the healing process. ${ }^{63}$ However, the assessment of bone healing via conventional radiographic methods is subjective and is less sensitive in predicting signs of early healing complications. ${ }^{8}$ Bone-biomarkers are the products of bone cell activity and are associated with several stages of bone healing. Consequently, bone-biomarkers have been analyzed in many of the reviewed in vivo studies for monitoring the process of bone regeneration, and provided an early diagnostic value for possible complications. ${ }^{64}$

Bone healing in response to implanted biomaterials is expected to proceed in three overlapping stages: early bone cell reactions, bone matrix deposition, and bone mineralization. ${ }^{11}$ The cellular interaction phase begins immediately after the implantation of biomaterials, which causes initial tissue damage and inflammation for approximately 3 to 4 days. There is evidence of formation of a fibrin-rich clot which acts as a scaffold for different molecular and cellular interactions which mediate angiogenesis. Subsequently, resorption of damaged bone by osteoclasts is observed as a key initiator for the stage of bone formation. ${ }^{9}$ The most specific and sensitive biomarker produced by bone resorbing osteoclasts is TRAP. ${ }^{65} \mathrm{Few}$ other biomarkers of osteoclastic activity include the RANKL and its membrane-bound receptor RANK and OPG, wherein bone resorption is inhibited by OPG when it binds to RANKL. ${ }^{66}$ Therefore, the balance between OPG and RANKL primarily regulates osteoclastic activity. ${ }^{67}$ In the present systematic review, 12 studies reported that TRAP, OPG, ALP, proliferating cell nuclear antigen (PCNA), BMP-2, BMP-3, BMP-4, COL-1, OPN, RUNX-2, and CBFA-1 were associated with the early stage of cellular response to biomaterials (Table 2). Interestingly, in 4 animal studies ${ }^{24,25,53,55}$ TRAP significantly correlated with osteoclast-like activity wherein TRAP5b was detected early in the postoperative immunoassays.

\section{Early Bone Cell Reactions}

Bone formation, when assessed at an early stage, not only has significant prognostic value, but could also facilitate confirmation of clinical success as reported by Prati et al. ${ }^{18}$ Alkaline phosphatase is an ubiquitous, membrane-bound tetrameric enzyme, commensurate with active remodeling of bone and is validated as a predictive indicator (Table 2) in majority of the reviewed literature. ${ }^{22,24,34,37,57,58}$ While Kabashima and Nagata ${ }^{20}$ found ALP to be associated with fibroblast-like regeneration, Sela et $\mathrm{al}^{43}$ demonstrated the peak expression of ALP between 14 and 21 days correlating with primary mineralization in newly formed bone surrounding titanium DI. Furthermore, bone anabolic activity signaled by FGFR through concurrent activation of RUNX-2 and BMP-2 was also reported. ${ }^{24}$ Few other biomarkers reportedly expressed in association with osteoblastic reactions include BALP, OCN, PINP, and COL-1. ${ }^{65}$

\section{Bone Matrix Deposition}

Bone matrix deposition is evident with the proliferation and differentiation of mesenchymal stem cells into osteoprogenitor cells and subsequently into osteoblasts. Bone formation markers are derived from osteoblasts, mainly during osteoid (bone matrix) synthesis (Table 2). The OCN plays an important role in ECM formation and osteoid mineralization through a negative feedback mechanism. An elevated serum level of OCN has been found during periods of rapid bone turnover and it has therefore been considered a valid biomarker when bone resorption and formation are coupled. ${ }^{10}$ Similarly, higher concentrations of OPN were observed in areas of bone formation with simultaneous recruitment and stimulation of macrophages and lymphocytes. Tera Tde et $\mathrm{al}^{28}$ reported intense bone metabolism associated with increased levels of OCN and OPN in healing osseous defect sites treated with e-PTFE and onlay bone graft. 
As one of the most abundant types of collagen in osseous tissue, COL-1 constitutes $90 \%$ of the organic matrix. The multitude of osteolytic changes occurring in bone remodeling and collagen degradation facilitates identification of COL-1 as a valuable biomarker of bone turnover. Two of the reviewed studies ${ }^{29,58}$ reported enhanced expression of COL-1 with a dose-dependent increase in matrix mineralization. The carboxy-terminal cross-linked telopeptides of COL-1 is not reused during collagen synthesis and are therefore considered as specific markers for bone resorption. ${ }^{45,53-55,57}$ During the intermediate stage of bone healing, OCN, COL-1, BSP, OPN, ONC, CD31+, BMP-2 and 7, VEGF, CBFA-1, and osterix (OSX) biomarkers were reportedly identified by 14 studies in the present review.

\section{Bone Mineralization}

Approximately 2 weeks following the initial implantation, osteoblasts deposit more woven (matrix) bone within the defect. ${ }^{68}$ Nevertheless, bone mineralization/remodeling starts only after adaptation of the morphology of new bone to the original tissue. For bone mineralization, 8 of the reviewed studies reported positive for OCN, OPN, CD31+, Ki-67, caspase-3, and TUNEL biomarkers (Table 2). Therefore, it would be alluring to assume impaired bone healing processes, associated with abnormal expression of these biomarkers. ${ }^{3}$ Reis-Filho et $\mathrm{al}^{33}$ and Kunert-Keil et $\mathrm{al}^{34}$ reported enhanced VEGF and VEGF-R2 expression correlating with evidence of angiogenesis in the target bone. In contrast, Tanaka et al ${ }^{40}$ reported a relatively lower expression of VEGF in new bone formation with GBR after a 10-day follow-up period. Based on the above reviewed studies, while VEGF elicited a chemo attractive effect on primary human osteoblasts and mesenchymal progenitor cells, it was significantly expressed only during the terminal stages of bone formation, preceded by an initial low level of detection. A high level of heterogeneity, possibly attributable to different experimental biomaterials, was observed in the reported expression of biomarkers associated with bone mineralization, which included PCNA, BMP, PINP, RANK, RANKL, TGF, Ki-67, Caspase-3, TUNEL and CD31.

\section{Subject Variability}

A variety of translational experimental models were used in the reviewed researches including dogs, sheep, rats, mice and rabbits, with each model considered to be ideal and simulating clinical scenarios. While, canine and sheep models exhibited maximum similarity in terms of outcomes measured, majority of the reviewed literatures were based on rodent models (rats and mice). Similarly, several types of bone defects desirable for mimicking bone regeneration in human bone were reported in the review. Interestingly, most of the studies which employed a craniofacial defect model in the mandible ${ }^{27-29,32,33,48,60}$ and the calvarial bone, ${ }^{22,24,34-36}$ reported appreciable bone formation and isolation of biomarkers for assessment. The variability of the physical and chemical characteristics of scaffolds, reported in the present review, also had a proven influence on bone regeneration and the related expression of biomarkers. For instance, subcutaneous pockets transplanted with collagen sponge and stem cells ${ }^{56}$ reported minimal bone formation, in contrast to defect sites treated with osteoconductive scaffolds. Although the efficacy of bone regeneration can be effectively evaluated by biomarkers of bone turnover, their prognostic importance needs to be substantiated. Similarly, the quantification of bone-biomarkers for studying bone metabolism through IHC assays lacks credible evidence in large-scale population studies, in spite of being comparatively less invasive and cost-effective. ${ }^{69}$ Nevertheless, clinical limitations in the use of biomarkers as standardized prognostic tools require continued development in identifying and quantifying more reliable biomarkers of bone healing. ${ }^{70}$

\section{Strengths and Limitations}

The major strengths of the present literature review are the systematic search strategy and adherence to PRISMA guidelines. However, a language bias may have influenced the study results, as only English language articles were included in this review. Nevertheless, this systematic review provides valuable insights of bone-biomarkers as prognostic indicators of bone regeneration and healing. The pronounced variability and heterogeneity of bonebiomarkers make it difficult to determine their precise thresholds and hence more observational studies are needed to be carried out to identify the desirable biomarkers. Further validation of biomarkers as independent determinants of bone turn over can be established only with long-term clinical studies.

\section{CONCLUSION}

Knowledge of bone biology and their regenerative potential has greatly expanded with advances in molecular biology and research. Major limitations of the scrutinized studies were related to the biological and analytical variability. In this review, several biomarkers were confirmed to be useful for the assessment of bone regeneration and healing around biomaterials. However, there was insufficient evidence to determine whether or not bonebiomarkers can be independently utilized to monitor bone regeneration around biomaterials. Nevertheless, standardization of analytical methods and formalizing 
protocols toward specific dominant bone-biomarkers can facilitate future research.

\section{ACKNOWLEDGMENTS}

The authors would like to thank the Deanship of Scientific Research and College of Dentistry Research Center (CDRC) at King Saud University, Riyadh, Saudi Arabia, for their help and support.

\section{REFERENCES}

1. Karring T, Nyman S, Gottlow J, Laurell L. Development of the biological concept of guided tissue regeneration - animal and human studies. Periodontol 20001993 Feb;1(1):26-35.

2. Retzepi M, Donos N. Guided Bone Regeneration: biological principle and therapeutic applications. Clin Oral Implants Res 2010 Jun;21(6):567-576.

3. Zimmermann G, Henle P, Kusswetter M, Moghaddam A, Wentzensen A, Richter W, Weiss S. TGF-beta1 as a marker of delayed fracture healing. Bone 2005 May;36(5):779-785.

4. Wang FS, Yang KD, Kuo YR, Wang CJ, Sheen-Chen SM, Huang $\mathrm{HC}$, Chen YJ. Temporal and spatial expression of bone morphogenetic proteins in extracorporeal shock wave-promoted healing of segmental defect. Bone 2003 Apr;32(4):387-396.

5. Sculean A, Nikolidakis D, Schwarz F. Regeneration of periodontal tissues: combinations of barrier membranes and grafting materials - biological foundation and preclinical evidence: a systematic review. J Clin Periodontol 2008 Sep;35 (8 Suppl):106-116.

6. Blanco J, Alonso A, Sanz M. Long-term results and survival rate of implants treated with guided bone regeneration: a 5 -year case series prospective study. Clin Oral Implants Res 2005 Jun;16(3):294-301.

7. Farina R, Bressan E, Taut A, Cucchi A, Trombelli L. Plasma rich in growth factors in human extraction sockets: a radiographic and histomorphometric study on early bone deposition. Clin Oral Implants Res 2013 Dec;24(12):1360-1368.

8. Bishop JA, Palanca AA, Bellino MJ, Lowenberg DW. Assessment of compromised fracture healing. J Am Acad Orthop Surg 2012 May;20(5):273-282.

9. Ram VS, Parthiban, Sudhakar U, Mithradas N, Prabhakar R. Bonebiomarkers in periodontal disease: a review article. J Clin Diagn Res 2015 Jan;9(1):ZE07-ZE10.

10. Kunimatsu K, Mataki S, Tanaka H, Mine N, Kiyoki M, Hosoda K, Kato Y, Kato I. A cross-sectional study on osteocalcin levels in gingival crevicular fluid from periodontal patients. J Periodontol 1993 Sep;64(9):865-869.

11. Leeming DJ, Alexandersen P, Karsdal MA, Qvist P, Schaller S, Tanko LB. An update on biomarkers of bone turnover and their utility in biomedical research and clinical practice. Eur J Clin Pharmacol 2006 Oct;62(10):781-792.

12. Farina R, Trombelli L. Wound healing of extraction sockets. Endodontic Topics 2011 Sep;25(1):16-43.

13. Cox G, Einhorn TA, Tzioupis C, Giannoudis PV. Boneturnover markers in fracture healing. Bone Joint J Surg Br 2010 Mar;92(3):329-334.

14. Konopka L, Pietrzak A, Brzezinska-Blaszczyk E. Effect of scaling and root planing on interleukin-1beta, interleukin- 8 and MMP-8 levels in gingival crevicular fluid from chronic periodontitis patients. J Periodont Res 2012 Dec;47(6):681-688.
15. Sexton WM, Lin Y, Kryscio RJ, Dawson DR 3rd, Ebersole JL, Miller CS. Salivary biomarkers of periodontal disease in response to treatment. J Clin Periodontol 2011 May;38(5): 434-441.

16. Leenaars $M$, Hooijmans CR, van VeggelN, terRietG, Leeflang $M$, Hooft L, van der Wilt GJ, Tillema A, Ritskes-Hoitinga M. A step-by-step guide to systematically identify all relevant animal studies. Lab Anim 2012 Jan;46(1):24-31.

17. Higgins, JP.; Green, S. editors. Cochrane handbook for systematic reviews of interventions. West Sussex: John Wiley \& Sons; 2011.

18. Prati AJ, Casati MZ, Ribeiro FV, Cirano FR, Pastore GP, Pimentel SP, Casarin RC. Release of bone markers in immediately loaded and nonloaded dental implants: a randomized clinical trial. J Dent Res 2013 Dec;92(12 Suppl):161S-167S.

19. Schulze-Spate U, Dietrich T, Kayal RA, Hasturk H, Dobeck J, Skobe Z, Dibart S. Analysis of bone formation after sinus augmentation using beta-tricalcium phosphate. Compend Contin Educ Dent 2012 May;33(5):364-368.

20. Kabashima H, Nagata K. Presence of interleukin-4-producing cells for human bone regeneration after application of guided tissue regeneration membranes. J Endod 2001 Jul;27(7):444-448.

21. Lima LL, Goncalves PF, Sallum EA, Casati MZ, Nociti FH Jr. Guided tissue regeneration may modulate gene expression in periodontal intrabony defects: a human study. J Periodontal Res 2008 Aug;43(4):459-464.

22. Aghaloo T, Cowan CM, Chou YF, Zhang X, Lee H, Miao S, Hong N, Kuroda S, Wu B, Ting K, et al. Nell-1-induced bone regeneration in calvarial defects. Am J Pathol 2006 Sep;169(3):903-915.

23. Tera Tde M, Nascimento RD, Prado RF, Santamaria MP, Jardini MA. Immunolocalization of markers for bone formation during guided bone regeneration in osteopenic rats. J Appl Oral Sci 2014 Nov-Dec;22(6):541-553.

24. Kodama N, Nagata M, Tabata Y, Ozeki M, Ninomiya T, Takagi R. A local bone anabolic effect of rhFGF2-impregnated gelatin hydrogel by promoting cell proliferation and coordinating osteoblastic differentiation. Bone 2009 Apr;44(4):699-707.

25. Taut AD, Jin Q, Chung JH, Galindo-Moreno P, Yi ES, Sugai JV, Ke HZ, Liu M, Giannobile WV. Sclerostin antibody stimulates bone regeneration after experimental periodontitis. J Bone Miner Res 2013 Nov;28(11):2347-2356.

26. Adeyemo WL, Reuther T, Bloch W, Korkmaz Y, Fischer JH, Zöller JE, Kuebler AC. Influence of host periosteum and recipient bed perforation on the healing of onlay mandibular bone graft: an experimental pilot study in the sheep. Oral Maxillofac Surg 2008 May;12(1):19-28.

27. Moreira DC, Sa CN, Andrade MG, Bório dos Santos Calmon de Bittencourt TC, de Almeida Reis SR, Pithon MM, Sadigursky M. Angiogenesis and osteogenesis at incorporation process of onlay bone graft. J Oral Maxillofac Surg 2013 Dec;71(12):2048-2057.

28. Tera Tde M, Prado RF, De Marco AC, Santamaria MP, Jardini MA. The RANK/RANKL/OPG interaction in the repair of autogenous bone grafts in female rats with estrogen deficiency. Braz Oral Res 2014 Sep;28(1):1-9.

29. Osugi M, Katagiri W, Yoshimi R, Inukai T, Hibi H, Ueda M. Conditioned media from mesenchymal stem cells enhanced bone regeneration in rat calvarial bone defects. Tissue Eng Part A 2012 Jul;18(13-14):1479-1489. 
30. Colombo JS, Balani D, Sloan AJ, Crean SJ, Okazaki J, Waddington RJ. Delayed osteoblast differentiation and altered inflammatory response around implants placed in incisor sockets of type 2 diabetic rats. Clin Oral Implants Res 2011 Jun;22(6):578-586.

31. Colombo JS, Satoshi S, Okazaki J, Crean SJ, Sloan AJ, Waddington RJ. In vivo monitoring of the bone healing process around different titanium alloy implant surfaces placed into fresh extraction sockets. J Dent 2012 Apr;40(4):338-346.

32. Jiang X, Zhao J, Wang S, Sun X, Zhang X, Chen J, Kaplan DL, Zhang Z. Mandibular repair in rats with premineralized silk scaffolds and BMP-2-modified bMSCs. Biomaterials 2009 Sep;30(27):4522-4532.

33. Reis-Filho CR, Silva ER, Martins AB, Pessoa FF, Gomes PV, de Araújo MS, Miziara MN, Alves JB. Demineralised human dentine matrix stimulates the expression of VEGF and accelerates the bone repair in tooth sockets of rats. Arch Oral Biol 2012 May;57(5):469-476.

34. Kunert-Keil C, Scholz F, Gedrange T, Gredes T. Comparative study of biphasic calcium phosphate with beta-tricalcium phosphate in rat cranial defects - a molecular-biological and histological study. Ann Anat 2015 May;199:79-84.

35. Pedersen TO, Xing Z, Finne-Wistrand A, Hellem S, Mustafa K. Hyperbaric oxygen stimulates vascularization and bone formation in rat calvarial defects. Int J Oral Maxillofac Surg 2013 Jul;42(7):907-914.

36. Kamakura S, Sasano Y, Homma H, Suzuki O, Kagayama M, Motegi K. Implantation of octacalcium phosphate nucleates isolated bone formation in rat skull defects. Oral Dis 2001 Jul;7(4):259-265.

37. Pati F, Song TH, Rijal G, Jang J, Kim SW, Cho DW. Ornamenting 3D printed scaffolds with cell-laid extracellular matrix for bone tissue regeneration. Biomaterials 2015 Jan;37: 230-241.

38. Lu K, Xu L, Xia L, Zhang Y, Zhang X, Kaplan DL, Jiang X, Zhang F. An ectopic study of apatite-coated silk fibroin scaffolds seeded with AdBMP-2-modified canine bMSCs. J Biomater Sci Polym Ed 2012 Jul;23(1-4):509-526.

39. Nomura I, Watanabe K, Matsubara H, Hayashi K, Sugimoto N, Tsuchiya H. Uncultured autogenous adipose-derived regenerative cells promote bone formation during distraction osteogenesis in rats. Clin Orthop Relat Res 2014 Dec;472(12):3798-3806.

40. Tanaka S, Matsuzaka K, Sato D, Inoue T. Characteristics of newly formed bone during guided bone regeneration: analysis of cbfa-1, osteocalcin, and VEGF expression. J Oral Implantol 2007 Feb;33(6):321-326.

41. Turri A, Elgali I, Vazirisani F, Johansson A, Emanuelsson L, Dahlin C, Thomsen P, Omar O. Guided bone regeneration is promoted by the molecular events in the membrane compartment. Biomaterials 2016 Apr;84:167-183.

42. Ratanavaraporn J, Furuya H, Kohara H, Tabata Y. Synergistic effects of the dual release of stromal cell-derived factor-1 and bone morphogenetic protein-2 from hydrogels on bone regeneration. Biomaterials 2011 Apr;32(11):2797-2811.

43. Sela J, Gross UM, Kohavi D, Shani J, Dean DD, Boyan BD, Schwartz Z. Primary mineralization at the surfaces of implants. Crit Rev Oral Biol Med 2000 Oct;11(4):423-436.

44. Du Z, Chen J, Yan F, Doan N, Ivanovski S, Xiao Y. Serum bone formation marker correlation with improved osseointegration in osteoporotic rats treated with simvastatin. Clin Oral Implants Res 2013 Apr;24(4):422-427.
45. Itala A, Valimaki VV, Kiviranta R, Ylänen HO,Hupa M, VuorioE, Aro HT. Molecular biologic comparison of new bone formation and resorption on microrough and smooth bioactive glass microspheres. J Biomed Mater Res Part B Appl Biomater 2003 Apr;65(1):163-170.

46. Samee M, Kasugai S, Kondo H, Ohya K, Shimokawa H, Kuroda S. Bone morphogenetic protein-2 (BMP-2) and vascular endothelial growth factor (VEGF) transfection to human periosteal cells enhances osteoblast differentiation and bone formation. J Pharmacol Sci 2008 Sep;108(1):18-31.

47. Liu HC, Ling-Ling E, Wang DS, Su F, Wu X, Shi ZP, Lv Y, Wang JZ. Reconstruction of alveolar bone defects using bone morphogenetic protein 2 mediated rabbit dental pulp stem cells seeded on nano-hydroxyapatite/collagen/poly(Llactide). Tissue Eng Part A 2011 Jun;17(19-20):2417-2433.

48. Marukawa K, Ueki K, Alam S, Shimada M, Nakagawa K, Yamamoto E. Expression of bone morphogenetic protein-2 and proliferating cell nuclear antigen during distraction osteogenesis in the mandible in rabbits. Br J Oral Maxillofac Surg 2006 Apr;44(2):141-145.

49. Alam S, Ueki K, Nakagawa K, Marukawa K, Hashiba Y, Yamamoto E, Sakulsak N, Iseki S. Statin-induced bone morphogenetic protein (BMP) 2 expression during bone regeneration: an immunohistochemical study. Oral Surg Oral Med Oral Pathol Oral Radiol Endod 2009 Jan;107(1):22-29.

50. TrejoCG,LozanoD,Manzano M,DoadrioJC,Salinas AJ,DapíaS, Gómez-Barrena E, Vallet-Regí M, García-Honduvilla N, Buján J, et al. The osteoinductive properties of mesoporous silicate coated with osteostatin in a rabbit femur cavity defect model. Biomaterials 2010 Nov;31(33):8564-8573.

51. Uchida R, Bhawal UK, Kiba H,AraiK, Tanimoto Y,KuboyamaN, Asakura T, Nishiyama N. Effect of plasma-irradiated silk fibroin in bone regeneration. J Biosci Bioeng 2014 Sep;118(3): 333-340.

52. Yoon SJ, Park KS, Kim MS, Rhee JM, Khang G, Lee HB. Repair of diaphyseal bone defects with calcitriol-loaded PLGA scaffolds and marrow stromal cells. Tissue Eng 2007 May;13(5):1125-1133.

53. Monjo M, Ramis JM, Ronold HJ, Taxt-Lamolle SF, Ellingsen JE, Lyngstadaas SP. Correlation between molecular signals and bone bonding to titanium implants. Clin Oral Implants Res 2013 Sep;24(9):1035-1043.

54. Monjo M, Lamolle SF, Lyngstadaas SP, Ronold HJ, Ellingsen JE. In vivo expression of osteogenic markers and bone mineral density at the surface of fluoride-modified titanium implants. Biomaterials 2008 Oct;29(28):3771-3780.

55. Wohlfahrt JC, Monjo M, Ronold HJ, Aass AM, Ellingsen JE, Lyngstadaas SP. Porous titanium granules promote bone healing and growth in rabbit tibia peri-implant osseous defects. Clin Oral Implants Res 2010 Feb;21(2):165-173.

56. Zhang W, Wang G, Liu Y, Zhao X, Zou D, Zhu C, Jin Y, Huang $Q$, Sun J, Liu X, et al. The synergistic effect of hierarchical micro/nano-topography and bioactive ions for enhanced osseointegration. Biomaterials 2013 Apr;34(13):3184-3195.

57. Rios CN, Skoracki RJ, Mathur AB. GNAS1 and PHD2 short-interfering RNA support bone regeneration in vitro and in an in vivo sheep model. Clin Orthop Relat Res 2012 Sep;470(9):2541-2553.

58. Barhanpurkar AP, Gupta N, Srivastava RK, Tomar GB, Naik SP, Joshi SR, Pote ST, Mishra GC, Wani MR. IL-3 promotes osteoblast differentiation and bone formation in human mesenchymal stem cells. Biochem Biophys Res Commun 2012 Feb;418(4):669-675. 
59. Tan J, Yang N, Fu X, Cui Y, Guo Q, Ma T, Yin X, Leng H, Song $C$. Single-dose local simvastatin injection improves implant fixation via increased angiogenesis and bone formation in an ovariectomized rat model. Med Sci Monit 2015 May;21:1428-1439.

60. Shirakura M, Fujii N, Ohnishi H, Taguchi Y, Ohshima H, Nomura S, Maeda T. Tissue response to titanium implantation in the rat maxilla, with special reference to the effects of surface conditions on bone formation. Clin Oral Implants Res 2003 Dec;14(6):687-696.

61. Guda T, Labella C, Chan R, Hale R. Quality of bone healing: perspectives and assessment techniques. Wound Repair Regen 2014 May;22(Suppl 1):39-49.

62. Adell R, Lekholm U, Rockler B, Brånemark PI. A 15-year study of osseointegrated implants in the treatment of the edentulous jaw. Int J Oral Surg 1981 Dec;10(6):387-416.

63. Zarb GA, Schmitt A. The longitudinal clinical effectiveness of osseointegrated dental implants: the Toronto study. Part III: problems and complications encountered. The J Prosthet Dent 1990 Aug;64(2):185-194.

64. Coulibaly MO, Sietsema DL, Burgers TA, Mason J, Williams BO, Jones CB. Recent advances in the use of serological bone formation markers to monitor callus development and fracture healing. Crit Rev Eukaryot Gene Expr 2010;20(2):105-127.
65. Cremers, S.; Garnero, P.; Seibel, MJ. Biochemical markers of bone metabolism. In: Bilezikian JP, Raisz LG, Martin TJ, editors. Principles of bone biology. 3rd ed. San Diego (CA): Academic Press; 2008. pp. 1857-1881.

66. Asagiri M, Takayanagi $\mathrm{H}$. The molecular understanding of osteoclast differentiation. Bone 2007 Feb;40(2):251-264.

67. Theoleyre S, Wittrant Y, Tat SK, Fortun Y, Redini F, Heymann D. The molecular triad OPG/RANK/RANKL: involvement in the orchestration of pathophysiological bone remodeling. Cytokine Growth Factor Rev 2004 Dec;15(6):457-475.

68. Esteves JC, Marcantonio E Jr, de Souza Faloni AP, Rocha FR, Marcantonio RA, Wilk K, Intini G. Dynamics of bone healing after osteotomy with piezosurgery or conventional drilling histomorphometrical, immunohistochemical, and molecular analysis. J Transl Med 2013 Sep;11:221.

69. Dabra S, China K, Kaushik A. Salivary enzymes as diagnostic markers for detection of gingival/periodontal disease and their correlation with the severity of the disease. J Indian Soc Periodontol 2012 Jul;16(3):358-364.

70. Florez-Moreno GA, Marin-Restrepo LM, Isaza-Guzman DM, Tobon-Arroyave SI. Screening for salivary levels of deoxypyridinoline and bone-specific alkaline phosphatase during orthodontic tooth movement: a pilot study. Eur J Orthod 2013 Jun;35(3):361-368. 